\title{
Pattern of Histopathological Alterations in N-methyl-N- Nitrosourea (MNU) Induced Breast Cancer in Female Sprague Dawley (SD) Rats Treated with Crude Honey
}

\author{
Urmila Banik $^{1,2 *}$, Sarfarz Ahamed ${ }^{3}$, Swe Swe Latt ${ }^{4}$, Nur Asyilla Che Jalil ${ }^{1}$, \\ Wan Faiziah Wan Abdul Rahman ${ }^{1}$ and Nor Hayati Othman ${ }^{1}$
}

${ }^{1}$ Department of Pathology, School of Medical Sciences, Universiti Sains Malaysia, 16150 USM, Kubang Kerian, Kelantan, Malaysia

${ }^{2}$ Unit of Pathology, Faculty of Medicine, AIMST University, Semeling, 08100 Bedong, Kedah, Malaysia

${ }^{3}$ Department of Biochemistry, Bahauddin Zakariya University, Multan, Pakistan

${ }^{4}$ Unit of Community Medicine, AIMST University, Faculty of Medicine, Semeling, 08100 Bedong, Kedah, Malaysia

\begin{abstract}
Natural products are becoming primary investigative molecules creating hope for finding new powerful classes of anticancer agents for breast cancer. One of the most remarkable of these is honey. To explore the mechanism of action of any anticancer agent the initial step is to analyse its effect in the histopathological tissue section. This study was designed to describe the histopathology of N-methyl$\mathrm{N}$-nitrosourea (MNU) induced breast cancer in Sprague Dawley rat (SD rats) treated with

ARTICLE INFO

Article history:

Received: 28 March 2020

Accepted: 15 June 2020

Published: 21 October 2020

DOI: https://doi.org/10.47836/pjst.28.4.22

E-mail addresses:

urmila_banik@yahoo.co.in; ub15_med074@student.usm.my (Urmila Banik)

sarfraz 114@yahoo.com (Sarfarz Ahamed)

sweswelattdr@gmail.com (Swe Swe Latt)

asyilla@usm.my (Nur Asyilla Che Jalil)

wfaiziahwar@gmail.com (Wan Faiziah Wan Abdul Rahman)

hayatikb@usm.my (Nor Hayati Othman)

*Corresponding author crude honey. Female rats were distributed into 4 groups: Group0 (normal), Group1 (MNU control), Group2 and 3: Tualang and Manuka honey-treated, respectively. Rats were sacrificed and histopathology of both non-treated and treated tumours was done. Lower histological grade, infrequent combination histologic pattern $(\mathrm{p}<0.001)$, prominent cytoplasmic vacuolization $(p<0.001)$, aggregates of atypical macrophages $(p<0.001)$ and lesser necrosis $(p=0.005)$ were
\end{abstract}


major outcomes in treated cancers. This data will aid in the histopathological interpretation of honey-treated breast cancer model and future anticancer study of honey.

Keywords: Breast cancer, histopathology, honey, MNU, Sprague Dawley rats

\section{INTRODUCTION}

Breast cancer is the most common cancer among women, affecting 2.1 million women every year and accounting for the highest number of cancer-related deaths among them (WHO, n.d.). According to traditional classification, primary breast adenocarcinoma is categorized as in situ and invasive. In situ cancers can be ductal carcinoma in situ (DCIS) or lobular (LCIS) and have a great prognosis. While $50-80 \%$ of the invasive cancers are invasive ductal carcinoma (IDC), only $25 \%$ of invasive breast cancers are 'special type' (invasive lobular carcinoma, invasive cribriform carcinoma and tubular carcinoma) (Masood, 2016). Histologic grading of invasive cancers is highly correlated with diseasefree and overall survival. Posttreatment histological alterations that are evident in breast cancer tissues are quite diverse. Histo-morphologically the Russo and Russo classification scheme for the N-methyl-N-nitrosourea (MNU) induced breast cancers in Sprague Dawley (SD) rats are quite alike to the traditional classification of human breast cancer (Russo \& Russo, 2000). This breast cancer model is simple to develop, cost-effective and mimics human breast cancer in terms of tumour histopathology, origination from mammary ductal epithelial cells and dependence on ovarian hormones for tumourigenesis. Hence it is a commonly used cancer model to study the anticancer mechanism of natural products and other potential anticancer agents (Han et al., 2019; Lu et al., 2013). However, cancers in rats may be composed of single histologic type or combinations of several patterns (Russo \& Russo, 2000).

Nowadays natural products are becoming the primary investigative molecules creating hope for finding new powerful classes of anticancer agents for breast cancer (Banik et al., 2017). One of the most remarkable natural compounds with substantial anticancer properties is honey. Polyphenols are the primary anticancer components of crude honey (Spilioti et al., 2014). Anticancer effect of honey is attributable to its anti-proliferative and pro-apoptotic activities (Jaganathan et al., 2015). Depending upon concentration crude honey can demonstrate either oestrogenic or antioestrogenic mode of action (Jaganathan \& Mandal, 2009). Although anticancer studies of honey on breast cancer primarily focuses on the molecular mechanistic effect of honey (Jaganathan et al., 2015), its detail mechanism of action is still indistinct. Generally, to explore the mechanism of action of any anticancer agent the initial step is to analyse its effect in the histopathological tissue section. Based on the histopathological observation subsequent suitable molecular methods are applied to understand the mechanism of action in detail. Precise correlation of post-treatment 
histopathological changes with underlying pathological processes helps to interpret the results accurately. Study shows that crude form of Tualang Honey (TH) and Manuka honey (MH) have a significant anticancer effect on breast cancer SD rat model (Ahmed et al., 2017). Our present study has been designed for the first time to do a detail analysis and comparison of the pattern of histological alterations in honey-treated MNU induced breast cancer in female SD rats with that of the non-treated ones.

\section{MATERIALS AND METHODS}

\section{Animals, MNU and Honey}

SD female rats (28 and 33 days old) were obtained from the Animal Research and Service Centre (ARASC), Universiti Sains Malaysia (USM). TH was supplied by the Federal Agricultural Marketing Authority (FAMA), Ministry of Agriculture and Agro based Industry, Malaysia. $\mathrm{MH}$ was purchased from the market (packed under license number 1003 for Vitaco Health (NZ) Ltd., New Zealand and imported and distributed by Cambert (M) Sdn.Bhd, Malaysia). MNU (Catalog number N1517-1G, Sigma, USA) and honey samples were prepared as described previously (Ahmed et al., 2017).

\section{Study Design}

Forty female SD rats were distributed into 4 groups (10 rats/group): Group0 (healthy normal control), Group1 (positive control, non-treated rats), Groups 2 and 3: 1g/kg body weight/ day of TH and MH was given orally, respectively. MNU was injected intraperitoneally ( $80 \mathrm{mg} / \mathrm{kg}$ body weight) into the rats around the age of 40 days. The rats in groups 1,2 and 3 were induced with cancer using carcinogen MNU. Mammary areas of the rats were palpated twice weekly to detect the appearance of masses. Oral honey treatment (using 1 $\mathrm{ml}$ syringes without needles administered to mouth) was started for groups 2 and 3 when the first palpable mass reached $10-12 \mathrm{~mm}$ size and continued till day $120 \mathrm{th}$. The rats were maintained on a standard balanced rat feed diet with water ad libitum and a $12 \mathrm{~h}$ day/ night cycle. On the day 120th, the rats were sacrificed following intraperitoneal injection of pentobarbital $100 \mathrm{mg} / \mathrm{kg}$ body weight. Tumour masses were examined in vivo, resected and fixed in neutral-buffered formalin. Subsequently, masses were paraffin-embedded, sectioned to $4 \mu$ thickness and stained with hematoxylin and eosin.

Total 39 non-treated and 36 honey-treated $(20 \mathrm{TH}, 16 \mathrm{MH})$ tissue sections were examined under a light microscope (Olympus Optical Co., Ltd., Tokyo, Japan). From the non-treated slide sections, 3 were excluded due to scanty tissue or poor slide quality. Russo and Russo guideline (Russo \& Russo, 2000) was followed for histological analysis. Grading was done by modified Bloom-Richardson system (Mukhopadhyay et al., 2006). Post-treatment alterations were analysed mainly by focusing on vacuolar degeneration, 
microcyst formation, tumour necrosis, tumour infiltrating lymphocytes and macrophages, hyalinization and fibrosis.

\section{Statistical Analysis}

Collected data was entered to an Excel sheet and transferred to and analysed with IBM SPSS version 20.0. The result of the descriptive statistics was shown as frequency and percentages. The chi-square test was used to analyse the relationship between outcome variables and non-treated \& treated groups with the p-value less than 0.05 as significant level.

\section{RESULTS AND DISCUSSION}

Total 103 breast cancer masses (positive control: 47, TH: 23 \& MH: 33) were collected from experimental animals. Grossly, tumour masses in non-treated control group were larger and harder as described previously (Ahmed et al., 2017). Both benign and malignant tumours were observed. Cancer masses in honey treated groups showed a lower median tumour size, weight, multiplicity compared with the non-treated control $(p<0.05)$. Honey treatment also demonstrated a dramatic slower growth rate (up to $70.82 \%$ ) compared with the nontreated control $(0 \%)(p<0.05)$. Routine histopathology was done. Total 72 histopathology slides (36 non-treated and 36 treated) were microscopically examined. All were malignant tumours of epithelial origin. In situ breast cancers were found in both non-treated control group $(n=3)$ and honey-treated group $(n=2)$. All were of single histologic pattern.

Among the non-treated invasive cancers ( $n=33$ ), a combination of $\geq 2$ patterns were frequently detected $(57.6 \%)$ (Table 1$)$. Histologic patterns observed in these invasive cancers were: papillary, cribriform, tubular and occasionally comedocarcinoma. However, no IDCs were seen. Papillary combined with cribriform was the most frequently $(30 \%)$ observed combination pattern (Table 2). The histologic grading ranged from grade 2 $(51.5 \%)$ to $3(48.5 \%)$.

Table 1

Histological types of breast cancers seen in non-treated control and honey treated female SD rats

\begin{tabular}{lllllll}
\hline \multirow{2}{*}{$\begin{array}{l}\text { Types of } \\
\text { breast } \\
\text { cancer }\end{array}$} & Histologic Findings & \multicolumn{3}{l}{ Non-treated control } & Honey-treated & p-value \\
\cline { 3 - 6 } & & Number & $\%$ & Number & $\%$ & \\
\hline In situ & Single histologic pattern & 3 & 60.0 & 2 & 40.0 & $\mathrm{p}=0.643$ \\
\hline Invasive & $\begin{array}{l}\text { Single histologic pattern } \\
\begin{array}{l}\text { Combination of } \geq 2 \\
\text { histologic patterns }\end{array}\end{array}$ & 14 & 32.6 & 29 & 67.4 & $\mathrm{p}<0.001$ \\
& & 79.2 & 5 & 20.8 & $\mathrm{p}<0.001$ \\
\hline
\end{tabular}


Table 2

Distribution of different combinations of histologic patterns of invasive breast cancers seen in the non-treated control and honey treated cancers of female SD rats

\begin{tabular}{|c|c|c|c|}
\hline \multicolumn{4}{|l|}{ Breast tissue of invasive breast cancer } \\
\hline $\begin{array}{l}\text { Histologic patterns observed in } \\
\text { Non-treated control }\end{array}$ & Number & $\begin{array}{l}\text { Histologic patterns observed } \\
\text { in Honey-treated }\end{array}$ & Number \\
\hline Papillary+Cribriform & 10 & Papillary + Cribriform & 4 \\
\hline $\begin{array}{l}\text { Papillary }+ \text { Cribriform }+ \text { Comedocarc } \\
\text { inoma }\end{array}$ & 1 & $\begin{array}{l}\text { Papillary }+ \text { Cribriform }+ \text { Comed } \\
\text { ocarcinoma }\end{array}$ & 1 \\
\hline Cribriform & 4 & Cribriform & 6 \\
\hline Cribriform+Tubular & 3 & - & - \\
\hline Papillary+ Tubular & 2 & - & - \\
\hline Tubular & 5 & Tubular & 4 \\
\hline Papillary & 5 & Papillary & 10 \\
\hline Papillary+Cribriform+Tubular & 1 & - & - \\
\hline Tubular+Comedocarcinoma & 1 & - & - \\
\hline Tubular+Comedocarcinoma & 1 & Tubular+Comedocarcinoma & 1 \\
\hline- & - & IDC & 8 \\
\hline- & - & Comedocarcinoma & 1 \\
\hline Total & 33 & & 34 \\
\hline
\end{tabular}

The degenerative features seen in the non-treated cancers $(n=36)$ were microcyst formation (39\%), hyalinization (75\%) and fibrosis (72\%) (Figure 1B-1D \& 1G-1I; Table 3). While hyalinization was more frequent in the nontreated group, fibrosis was slightly common in the treated ones. However, microcyst formation was equivalent in both the groups. Vacuolar degeneration with tumour cell swelling was quite significant in the treated cancers in comparison to the non-treated ones $(\mathrm{p}<0.001)$. Eighty-nine percent of the treated cancers showed vacuolar degeneration. Swollen cancer cells coalesced to form cystic spaces followed by disintegration. In the non-treated controls, this vacuolar degeneration was very mild or absent (Figure 1E, 1J; Table 3).

In the non-treated cancers mild to moderate tumour infiltrating lymphocytes (TIL) with or without aggregates of foamy large a typical macrophages and eosinophil were frequently seen. Likewise, in treated cancers TILs were associated with tumour associated aggregates of foamy large atypical macrophages. Interestingly although in the treated group these a typical bizarre-looking macrophages with large hyperchromatic nucleus were seen in many of the cancers $(71.4 \%)$, compared with the non-treated cancers, they were somewhat inconspicuous (Figure 1F, 1K; Table 3). Mild to massive tumour necrosis (65.8\%) were 
noted in the non-treated control cancers (Table 3). However, in the treated ones, necrosis was present in $34.2 \%$ of cases with massive necrosis seen in only $5.55 \%$ cases.

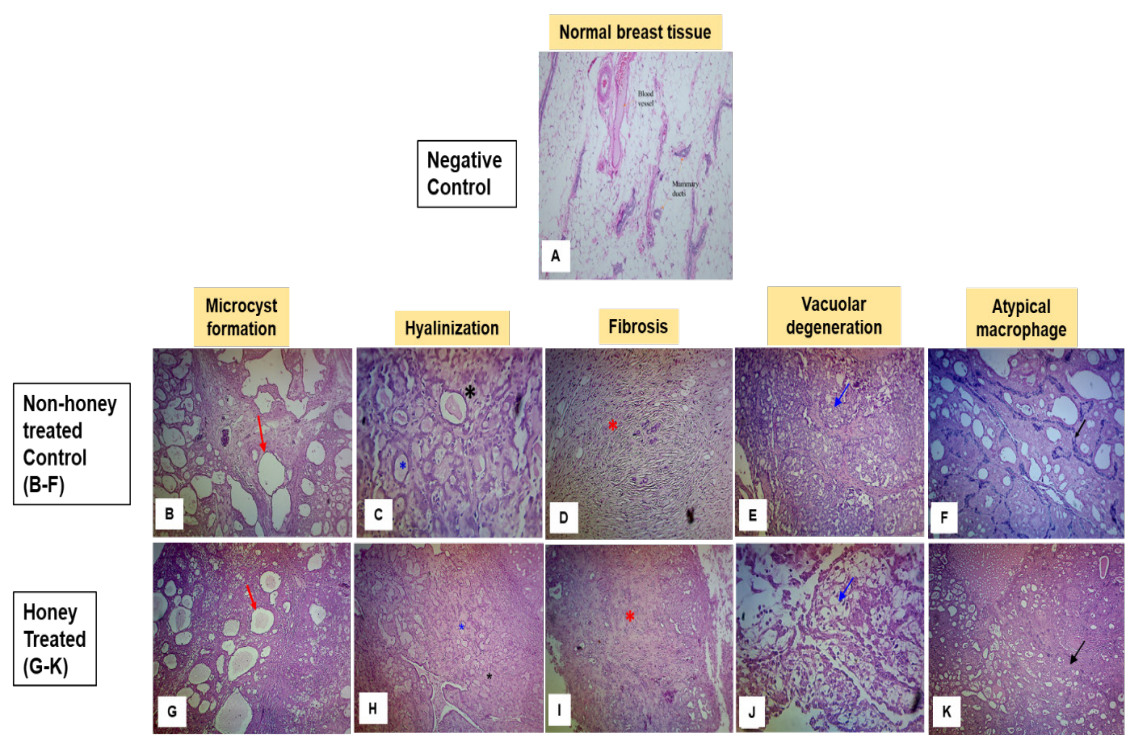

Figure 1. Histopathological findings of honey-treated and non-treated control breast cancer in female SD rats (200X magnification). (A): Histology of normal mammary gland of female Sprague-Dawley rat. Mammary ducts are surrounded by adipose and fibrous tissue with the varied distribution. H\&E staining magnification x100. $(B, G)$ : Microcyst formation was equivalent in both nontreated control and honey treated group (Red arrow). (C, H): Hyaline material surrounding nests of malignant cells; extraluminal hyaline (Black asterisk): the intraluminal hyaline (Blue asterisk) is due to the secretory material. (D, I): Small nests of malignant cells surrounded by dense fibrous tissue (Red asterisk) deposition. (E, J): Cellular swelling (Blue arrow) was more prominent in the treated group. Absent or mild cytoplasmic vacuolization was seen in the nontreated control group. $(\mathrm{F}, \mathrm{K})$ : Bizarre atypical macrophages in tumour parenchyma and tumour stroma of both honey-treated and nontreated control cancers (Black arrow). These cells are more prominent in the control group.

Table 3

Histomorphological alterations seen in non-treated control and honey treated breast cancers

\begin{tabular}{|c|c|c|c|c|c|}
\hline \multirow[b]{2}{*}{ Traits } & \multicolumn{2}{|c|}{ Non-treated control } & \multicolumn{2}{|c|}{ Honey treated } & \multirow[t]{2}{*}{ p-value } \\
\hline & Number & $\%$ & Number & $\%$ & \\
\hline Microcyst & 14 & 50.0 & 14 & 50.0 & $\mathrm{p}>0.99$ \\
\hline Hyalinization & 27 & 60.0 & 18 & 40.0 & $\mathrm{p}=0.028$ \\
\hline Fibrosis & 26 & 46.4 & 30 & 53.6 & $\mathrm{p}=0.257$ \\
\hline Vacuolar degeneration & 2 & 5.9 & 32 & 94.1 & $\mathrm{p}<0.001$ \\
\hline Tumor necrosis & 25 & 65.8 & 13 & 34.2 & $\mathrm{p}=0.005$ \\
\hline Foamy macrophages & 12 & 28.6 & 30 & 71.4 & $\mathrm{p}<.001$ \\
\hline
\end{tabular}


Post-treatment histopathological changes in tumour morphology play an important role in evaluating the therapeutic response. With increasingly accumulating data on distinct molecular-morphologic correlates, there is a resurgence of attention in the role of tissue evaluation in in vivo cancer study. In breast cancer, diverse histopathologic alterations are observed with the application of different therapeutic modalities. With radiation therapy, bizarre atypical nuclear changes and abnormal mitotic figures are usually seen. Yet again widespread tumour necrosis may develop and become bounded by fibrosis (Schnitt et al., 1984). Hormone therapy can cause connective tissue deposition with striking stromal fibrosis, hyalinization. Cytoplasmic vacuolization, cell membrane disruption, nuclear aberrations, and eventual necrosis are frequent changes seen in post endocrine therapy (Kennedy et al., 1990). Chemotherapy can induce vacuolar degeneration in the cancer cells. Neoadjuvant chemotherapy does not usually affect the histologic grading of the carcinoma (Kennedy et al., 1990). Our study showed that honey treatment led to various degenerative changes in breast cancer tissue. These are mainly due to cellular response to the injurious effect of crude honey on cancer cells. These histologic changes could imply good prognosis. In breast cancer tumour grading is more clinically significant than the number and type of patterns observed. Histologic grading involves identification of cellular pleomorphism, tubular formation and mitoses of the breast cancer cells. Nevertheless, the tumour histologic patterns may also have some impact on the prognosis.

Cytoplasmic vacuolization is a well-recognized morphological alteration seen in mammalian cells after exposure to many pathogens and different natural compounds (Shubin et al., 2016). It often accompanies cell death (Bouzas-Rodríguez et al., 2012; Li et al., 2013; Shubin et al., 2016; Singha et al., 2013). A portion of the inducers of irreversible vacuolization causes known types of caspase-independent cell death (Christofferson \& Yuan, 2010; Overmeyer et al., 2008; Weerasinghe \& Buja, 2012). Our present study indicates that cellular vacuolization is a characteristic outcome observed in the histopathology of crude honey-treated MNU induced breast cancer in female SD rats. This indicates that crude honey has a cytotoxic effect on the breast cancer cells and vacuoles can be a side effect of the action of cytotoxic factors in crude honey. It is important to correlate this alteration with the effect of honey on the cancer cell cycle and cell death.

Presence of necrosis indicates aggressiveness of a malignant tumour and implies a high proliferative rate of the cancer cells. Usually the higher the rate of tumour cells proliferation, the higher the occurrence of ischaemic necrosis in tumour tissue. Yet again post-treatment necrosis in a treated malignant tumour may indicate the positive response towards therapy. In our study, we found that the frequency of necrosis was less in the honey-treated group $(\mathrm{p}=0.005)$ compared with that of the control ones. It can be presumed that due to the growth inhibitory activity of honey, cancer cell proliferation drops and there is a lesser amount of ischemic necrosis. 
In early tumours, tumour associated macrophages appear to have an inflammatory, tumouricidal M1 phenotype. However, with tumour progression, these macrophages polarise towards M2-like phenotype that is protumour, angiogenic and immune-inhibitory (Fridlender \& Albelda, 2013). In the case of TILs, density and the phenotypic profile are important prognostic factors. Our study revealed that aggregates of macrophages were present in the stroma and parenchyma of both the non-treated and treated MNU induced breast cancers. However, these were less obvious in the treated ones. It would be interesting to analyse the phenotype of the lymphocytes and macrophages, especially in the treated cancers.

Among the broad range of breast cancer animal models, orthotopic xenograft models are cutting-edge cancer model for the evaluation of novel anticancer agents (Whittle et al., 2015). Patient-derived xenografts (PDXs) are not only expensive but also need many elaborate settings and requirements like fresh patient material, immunocompromised host. Furthermore, in PDX, mouse stroma replaces human with increasing passages (Holen et al., 2017). For our current study, we have used MNU induced SD rat breast cancer model as it is not only simple to develop but also cost-effective and addresses the specific research question and aim of our study quite effectively.

\section{CONCLUSION}

The present study is focused mainly on the various histomorphological alterations observed in MNU induced experimental breast cancer model in female SD rats after crude honey treatment. Crude honey has anti-cancer effect as seen histologically in this in vivo study. In this study for the first time, we have described the morphological alterations in MNU induced breast cancer in SD rats after crude honey treatment. Presence of single morphological pattern, lower histopathological grade, prominent cytoplasmic vacuolization in the cancer cells, frequent aggregates of tumour-associated atypical large macrophages with scanter necrosis and slightly augmented fibrosis were the major post-treatment outcomes observed. Lower histological grade of cancer with cytoplasmic vacuolisation in cancer cells and less necrosis implicates the positive efficacy of crude honey as an anticancer agent on breast cancer tissue. The overall post-therapeutic histomorphological alterations showed similarity with that of the post-therapeutic alterations caused by endocrine therapy and to some extent chemotherapy. These histopathological findings will direct towards a future anticancer study on honey and its derivatives. It will also help in molecular correlation and histological interpretation of honey-treated breast cancer tissue in the animal model. The histopathological parameters used in this study to describe the anticancer effect of honey in the animal model can be applied to evaluate the effect of other potential anticancer agents. 


\section{ACKNOWLEDGEMENT}

The project is supported by USM RUI Grant 1001 PPSP 8012299.

\section{REFERENCES}

Ahmed, S., Sulaiman, S. A., \& Othman, N. H. (2017). Oral administration of Tualang and Manuka honeys modulates breast cancer progression in Sprague-Dawley rats model. Evidence-Based Complementary and Alternative Medicine: ECAM, 2017, 1-15.

Banik, U., Parasuraman, S., Adhikary, A. K., \& Othman, N. H. (2017). Curcumin: The spicy modulator of breast carcinogenesis. Journal of Experimental and Clinical Cancer Research: CR, 36(1), 1-16.

Bouzas-Rodríguez, J., Zarraga-Granados, G., del Rayo Sanchez-Carbente, M., Rodríguez-Valentín, R., Gracida, X., Anell-Rendón, D., ... \& Castro-Obregón, S. (2012). The nuclear receptor NR4A1 induces a form of cell death dependent on autophagy in mammalian cells. PLoS One, 7(10), 1-12.

Christofferson, D. E., \& Yuan, J. (2010). Necroptosis as an alternative form of programmed cell death. Current Opinion in Cell Biology, 22(2), 263-268.

Fridlender, Z. G., \& Albelda, S. M. (2013). Modifying tumor-associated macrophages. Oncoimmunology, 2(12), 1-3.

Han, B., Peng, X., Cheng, D., Zhu, Y., Du, J., Li, J., \& Yu, X. (2019). Delphinidin suppresses breast carcinogenesis through the HOTAIR/microRNA-34a axis. Cancer Science, 110(10), 3089-3097.

Holen, I., Speirs, V., Morrissey, B., \& Blyth, K. (2017). In vivo models in breast cancer research: Progress, challenges and future directions. Disease Models and Mechanisms, 10(4), 359-371.

Jaganathan, S. K., Balaji, A., Vellayappan, M. V., Asokan, M. K., Subramanian, A. P., John, A. A., ... \& Marvibaigi, M. (2015). A review on antiproliferative and apoptotic activities of natural honey. Anti-Cancer Agents in Medicinal Chemistry, 15(1), 48-56.

Jaganathan, S. K., \& Mandal, M. (2009). Antiproliferative effects of honey and of its polyphenols: A review. Journal of Biomedicine and Biotechnology, 2009, 1-13.

Kennedy, S., Merino, M. J., Swain, S. M., \& Lippman, M. E. (1990). The effects of hormonal and chemotherapy on tumoral and nonneoplastic breast tissue. Human Pathology, 21(2), 192-198.

Li, X., Sui, C., Chen, Q., Chen, X., Zhang, H., \& Zhou, X. (2013). Promotion of autophagy at the maturation step by IL- 6 is associated with the sustained mitogen-activated protein kinase/extracellular signal-regulated kinase activity. Molecular and Cellular Biochemistry, 380(1), 219-227.

Lu, Y., You, M., Ghazoui, Z., Liu, P., Vedell, P. T., Wen, W., .. \& Lubet, R. A. (2013). Concordant effects of aromatase inhibitors on gene expression in ER+ Rat and human mammary cancers and modulation of the proteins coded by these genes an uninvited scientific article. Cancer Prevention Research, 6(11), 1151-1161. 
Masood, S. (2016). Breast cancer subtypes: Morphologic and biologic characterization. Women's Health, 12(1), 103-119.

Mukhopadhyay, S., Ballard, B. R., Mukherjee, S., Kabir, S. M., \& Das, S. K. (2006). Beneficial effects of soy protein in the initiation and progression against dimethylbenz [a] anthracene-induced breast tumors in female rats. Molecular and Cellular Biochemistry, 290(1-2), 169-176.

Overmeyer, J. H., Kaul, A., Johnson, E. E., \& Maltese, W. A. (2008). Active ras triggers death in glioblastoma cells through hyperstimulation of macropinocytosis. Molecular Cancer Research: MCR, 6(6), 965-977.

Russo, J., \& Russo, I. H. (2000). Atlas and histologic classification of tumors of the rat mammary gland. Journal of Mammary Gland Biology and Neoplasia, 5(2), 187-200.

Schnitt, S. J., Connolly, J. L., Harris, J. R., \& Cohen, R. B. (1984). Radiation-induced changes in the breast. Human Pathology, 15(6), 545-550.

Shubin, A. V., Demidyuk, I. V., Komissarov, A. A., Rafieva, L. M., \& Kostrov, S. V. (2016). Cytoplasmic vacuolization in cell death and survival. Oncotarget, 7(34), 55863-55889.

Singha, P. K., Pandeswara, S., Venkatachalam, M. A., \& Saikumar, P. (2013). Manumycin A inhibits triplenegative breast cancer growth through LC3-mediated cytoplasmic vacuolation death. Cell Death and Disease, 4(1), e457- e457.

Spilioti, E., Jaakkola, M., Tolonen, T., Lipponen, M., Virtanen, V., Chinou, I., ... \& Moutsatsou, P. (2014). Phenolic acid composition, antiatherogenic and anticancer potential of honeys derived from various regions in Greece. PLOS ONE, 9(4), 1-10.

Weerasinghe, P., \& Buja, L. M. (2012). Oncosis: An important non-apoptotic mode of cell death. Experimental and Molecular Pathology, 93(3), 302-308.

Whittle, J. R., Lewis, M. T., Lindeman, G. J., \& Visvader, J. E. (2015). Patient-derived xenograft models of breast cancer and their predictive power. Breast Cancer Research: BCR, 17(1), 1-13.

WHO. (n.d.).| Breast cancer. World Health Organization. Retrieved August 14, 2019, from http://www.who. int/cancer/prevention/diagnosis-screening/breast-cancer/en/ 